\title{
An additional record of the non-indigenous species (NIS) Seriola fasciata from the southern coast of Sicily (Central Mediterranean Sea)
}

\author{
Michele Luca GERACI ${ }^{1,2}$, Fabio FALSONE ${ }^{2}$, Danilo SCANNELLA ${ }^{2}$ and Sergio VITALE ${ }^{2}$ \\ ${ }^{1}$ Department of Biological, Geological and Environmental Sciences (BiGeA) - Marine biology \\ and fisheries laboratory of Fano (PU), University of Bologna (BO) \\ ${ }^{2}$ Institute for Biological Resources and Marine Biotechnology (IRBIM), National Research \\ Council - CNR, Mazara del Vallo (Tp), Italy \\ Corresponding author, e-mail: fabio.falsone@irbim.cnr.it
}

An additional record of the non-indigenous species (NIS) Seriola fasciata from the southern coast of Sicily (Central Mediterranean Sea) is here described in this note.

The catch record is the first in the area and confirms the key role of the area for NIS spreading. In addition, an updated map of its spatial distribution is provided as well as a discussion on the possible misidentification and competition with the native greater amberjack Seriola dumerili.

Key words: Herculean species, non-indigenous species, biodiversity, spatial distribution, Strait of Sicily

\section{INTRODUCTION}

The biodiversity of the Mediterranean Sea has changed considerably over the past two decades, mainly due to the increasing occurrence of non-indigenous species (NIS) introduced both naturally (i.e. via Suez Canal and the Strait of Gibraltar) and due to anthropic activities, such as marine shipping (ballast waters, fouling) and aquaculture (GALIL et al., 2017). NIS are classified as lessepsian species, if migrate from the Red Sea to the eastern Mediterranean Sea across the Suez Canal, or herculean, if a species naturally via Strait of Gibraltar (GOULLETQUER et al., 2014; LOCKWOOD et al., 2013). Fishes are an important component of NIS and among them the majority are lessepsian species (DEIDUN et al., 2015).

The most successful lessepsian fish species are Fistularia commersonii (Rüppell, 1838) (AZZURRO et al., 2013; VITALE et al., 2016); Lagocephalus scleratus (Linnaeus, 1758) (AZZURRO et al., 2014; KARA et al., 2015); Pterois miles (J. W. Bennett, 1828) (VAVASIS et al., 2020); Siganus luridus (Rüppell, 1828) (AZZURRO \& ANDALORO, 2004); Stephanolepis diaspros (Fraser-Brunner, 1940) (DEIDUN et al., 2015) and Upeneus pori (Ben-Tuvia \& Golani, 1989) (DEIDUN et al., 2018; GERACI et al., 2018). Although herculean species represent the minority of NIS their settlement effects on Mediterranean marine communities are not to be neglected, especially if they compete with other species particularly important to the local economies. The Lesser Amberjack Seriola fasciata (Bloch, 1973) is a herculean species, widespread across the Atlantic Ocean that may compete with the native greater amber- 
jack Seriola dumerili (Risso, 1810). In the Mediterranean Sea, it was recorded for the first time in Balearic Island (MASSUTI \& STEFANESCU, 1993) and then has spread rapidly eastward up to Israel, Lebanon, Syrian and waters (SONIN et al., 2009; CROCETTA et al., 2015; JAWAD et al., 2015). The rapid natural expansion of the species via Strait of Gibraltar would be supported by an increase in water flux through the strait and hydroclimate modifications, such as temperature increase, which would favour the settlement of species of subtropical and tropical affinity (ANDALORO \& RINALDI, 1998; QUIGNARD \& TOMASINI, 2000). The present note concerns the first record of $S$. fasciata in the southern coast of Sicily and provides an update of its spatial distribution.

\section{MATERIAL AND METHODS}

In November 2016, off the southern coast of Sicily (Terrible Bank: approximate coordinates $37.15000^{\circ} \mathrm{N}, 12.88333^{\circ} \mathrm{E}$ ), one specimen of $S$. fasciata was caught using trammel net at about $50 \mathrm{~m}$ depth. It was identified according to the morphological description and meristic given by FISCHER et al. (1981) and GOLANI et al. (2002). In addition, the meristic and morphometric characteristics of the specimen were compared with other Mediterranean records (ANDALORO $e t$ al., 2005; JAWAD et al., 2015; STAMOULI et al., 2017; DOĞDU et al., 2019). In particular, only for ANDALORO et al. (2005) the morphometric features of nine specimens were provided as mean values. All measurements were performed through ImageJ software (RUEDEN et al., 2017) to the nearest $0.5 \mathrm{~cm}$ while sex was determined macroscopically. Then, maturity stage was determined through Medits (International bottom trawl in the Mediterranean) scale (Anon., 2017). Map of the S. fasciata first records in the Mediterranean was made by means Quantum GIS software in order to update its spatial distribution.

\section{RESULTS AND DISCUSSION}

Specimen was an immature male, measured $20.5 \mathrm{~cm} \mathrm{TL}$ and weighted $128.8 \mathrm{~g}$. The end of upper jaw relatively narrow, the typical irregu-

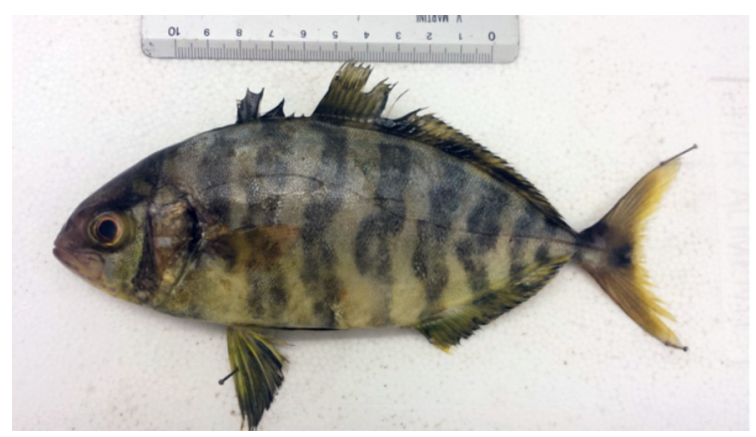

Fig. 1. Specimen of Seriola fasciata caught off the southern coast of Sicily

lar dark body bars and meristic count were in agreement with FISCHER et al. (1981) and GOLANI et al. (2002) (Fig.1). In addition, as shown in Table 1 by comparing the morphometric and meristic features it was emerged that all values were similar with the other Mediterranean studies which strengthen the correct identification of the species and therefore the presence of $S$. fasciata in Strait of Sicily. The occurrence of $S$. fasciata in the Sicilian waters is not totally unexpected indeed the Strait of Sicily may represents an ecological corridor for NIS from the Atlantic Ocean and the Indo-Pacific Ocean as well (SCANNELLA et al., 2017; SERVELLO et al. 2019, GERACI et al., 2019; FALSONE et al., 2020). Around Sicily waters, the catch of juveniles of S. fasciata with trammel net represents an anomaly considering that it was caught mainly caught by purse seine fishing the common dolphinfish (Coryphaena hippurus) with fish aggregating devices (FADs) (ANDALORO et al., 2005; TIRALONGO et al., 2018) (Fig. 2).

Concerning spatial distribution, in the Strait of Sicily S. fasciata was never reported in the southern coast of Sicily. Previously it has been reported both in Lampedusa (ANDALORO et al., 2005) and Malta islands (DEIDUN et al., 2011) while the closest finding to the present record was in Egadi Islands (LIPEJ et al., 2017) (Fig. 2). Despite GOLANI et al. (2002) stated that most of the Atlantic fish are irregular species and unable to create stable populations in their new habitats, $S$. fasciata represents a successful colonizer becoming a commercial species in central Mediterranean Sea (ANDALORO et al., 2005). It is worth to highlight as to date in the Mediterranean, only 


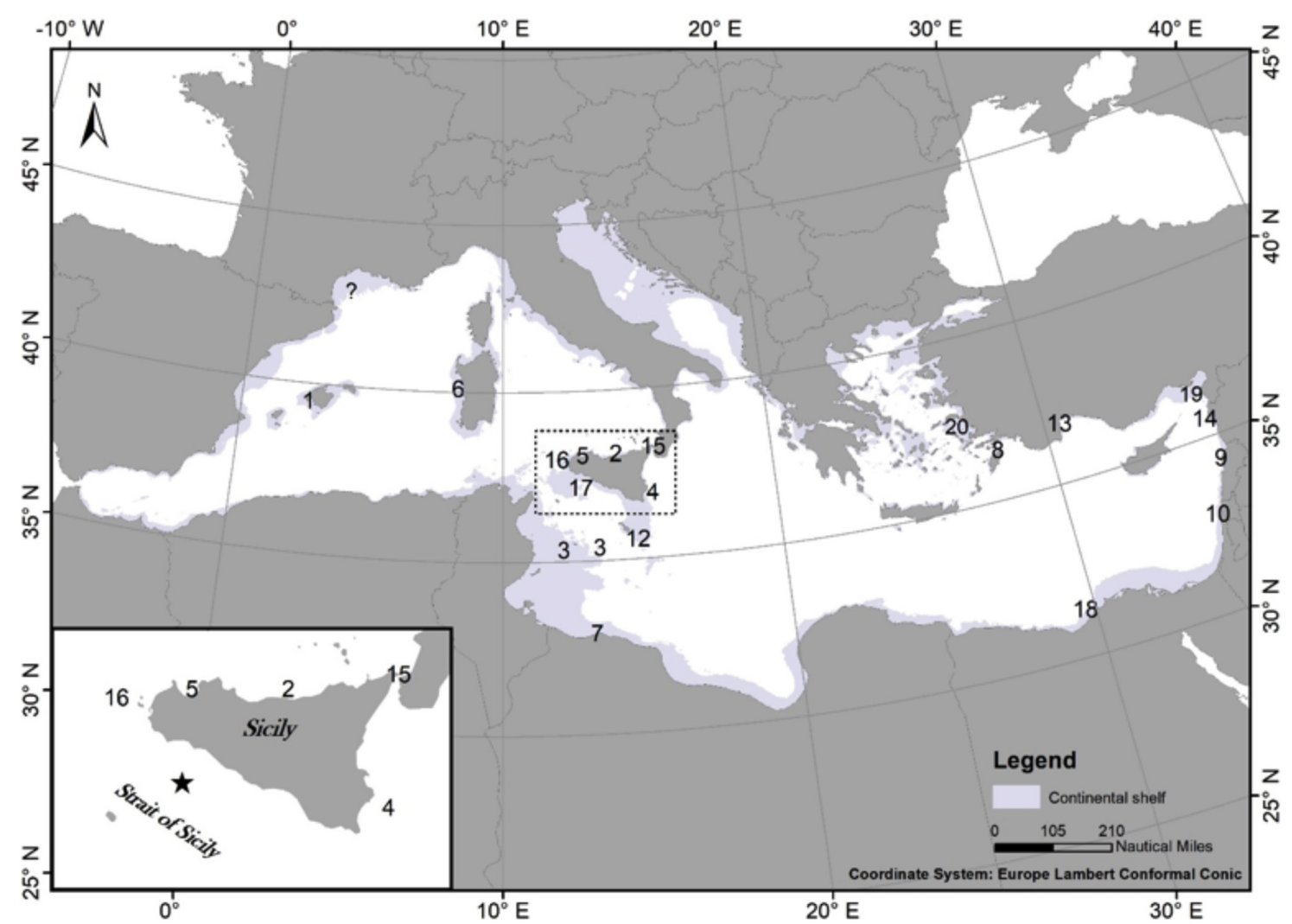

Fig. 2. Updated map showing location of Seriola fasciata first records in the Mediterranean basin reported according to the catch date. 1: 1989 (Massuti \& Stefanescu, 1993); 2, 3, 4, 5, 6: 1994-1995,1997-1998, 2002 (Andaloro et al., 2005); 7: 2003 (Shakman et al., 2017); 8: 2004 (Corsini et al., 2006); 9: 2005 (Crocetta \& Bariche, 2015); 10: 2008 (Sonin et al., 2009); 11, 12: 2008-2009 (Deidun et al., 2011); 13: 2012 (Özvarol \& Gökoğlu, 2014); 14: 2013 (Jawad et al., 2015); 15: 2014 (Castriota \& Spinelli, 2016); 16: 2016 (Castriota \& Falautano, 2017); 17 (black star): present study; 18: 2017 (Akel and Rizkalla, 2017);19: 2018 (Doğdu et al. 2019); 20: 2018 (Yapici \& Filiz, 2020); ?: doubtful record (Quignard, 1996, no published data)

a record above the $40^{\text {th }}$ parallel, i.e. Gulf of Lion, was reported by QUIGNARD \& TOMASINI, (2000) based on unpublished record (QUIGNARD, 1996, no published) (Fig. 2). This spatial pattern may be due to the subtropical and tropical affinity of the species that hampers its spreading toward the highest latitudes where the marine waters result generally colder.

Regarding morphological aspects, it should be noted that due to the similarity between the adults of $S$. dumerili and S. fasciata, fishers may confuse the two conspecific carangidae. As matter of fact, the only macroscopic difference of the above mentioned species is the supramaxilla, wide in $S$. dumerili narrow in $S$. fasciata (ISPRA, 2012). So, the presence and abundance of $S$. fasciata in the Mediterranean Sea might be underestimated (TIRALONGO et al., 2018). Indeed, most of the records concern juveniles, which are more easily identifiable than adults. The possible adult misidentification with $S$. dumerili lead to define the $S$. fasciata as cryptogenic questioning if the specie is really a NIS (CROCETTA et al., 2015). Indeed, the term cryptogenic refers to circumtropical species or species with a disjoint distribution, and whose native range is still unknown or its presence may be the result of past introductions not recorded in the literature (CARLTON, 1996). In the light of the above, further investigation will be needed to monitor this species expansion in the southern coast of the Mediterranean sea and better understand its possible competition with the greater amberjack S. dumerili. 
Table 1. Seriola fasciata morphometric and meristic characters of the present study (Strait of Sicily) compared with other Mediterranean Sea records

\begin{tabular}{|c|c|c|c|c|c|c|}
\hline $\begin{array}{l}\text { Morphometric and } \\
\text { meristic characters }\end{array}$ & $\begin{array}{l}\text { ANDALORO et } \\
a l ., 2005\end{array}$ & $\begin{array}{l}\text { JAWAD et } \\
\text { al., } 2015\end{array}$ & $\begin{array}{c}\text { STAMOULI et } \\
\text { al., } 2017\end{array}$ & $\begin{array}{l}\text { DOĞDU et al. } \\
2019\end{array}$ & $\begin{array}{c}\text { YAPICI } \\
\& \text { FILIZ, } \\
2020\end{array}$ & $\begin{array}{l}\text { Present } \\
\text { study }\end{array}$ \\
\hline Weight (g) & NA & NA & 49 & 106.2 & 587.5 & 128.8 \\
\hline \multirow[t]{2}{*}{ Total length (cm) } & 27.4 & 16.9 & 16 & 18.3 & 34.9 & 20.5 \\
\hline & & & $\%$ Total Length & & & \\
\hline Fork length & $86.8 \%$ & $87.6 \%$ & $87.5 \%$ & $87.4 \%$ & $86.24 \%$ & $88.9 \%$ \\
\hline Standard length & $82.8 \%$ & $83.4 \%$ & $77.5 \%$ & $76.5 \%$ & $75.64 \%$ & $84.5 \%$ \\
\hline $\begin{array}{l}\text { Pre 1st dorsal fin } \\
\text { length }\end{array}$ & $26.4 \%$ & $33.1 \%$ & $33.1 \%$ & $34.3 \%$ & $27.42 \%$ & $29.0 \%$ \\
\hline 1st dorsal fin length & $7.7 \%$ & $8.3 \%$ & $10.6 \%$ & $11.5 \%$ & $8.49 \%$ & $10.7 \%$ \\
\hline $\begin{array}{l}\text { 2nd dorsal fin } \\
\text { length }\end{array}$ & $37.4 \%$ & $36.7 \%$ & $36.2 \%$ & $37.7 \%$ & $34.84 \%$ & $37.8 \%$ \\
\hline $\begin{array}{l}\text { Pre second dorsal } \\
\text { fin length }\end{array}$ & NA & $42.0 \%$ & $41.3 \%$ & $40.7 \%$ & $27.42 \%$ & $40.8 \%$ \\
\hline $\begin{array}{l}\text { Height of } 2 \mathrm{nd} \\
\text { dorsal fin lobe }\end{array}$ & $13.5 \%$ & NA & $10.0 \%$ & $9.7 \%$ & NA & $10.7 \%$ \\
\hline Pre pectoral length & NA & NA & $26.3 \%$ & $24.3 \%$ & NA & $24.0 \%$ \\
\hline Pectoral length & $12.8 \%$ & NA & $10.6 \%$ & $14.4 \%$ & NA & $15.5 \%$ \\
\hline $\begin{array}{l}\text { Pre ventral fin } \\
\text { length }\end{array}$ & NA & NA & $26.9 \%$ & $30.7 \%$ & NA & $28.5 \%$ \\
\hline Ventral fin length & $19.5 \%$ & NA & $13.8 \%$ & $19.2 \%$ & NA & $19.7 \%$ \\
\hline Pre anal fin length & $44.8 \%$ & NA & $53.8 \%$ & $52.4 \%$ & $43.98 \%$ & $55.6 \%$ \\
\hline Anal fin length & $21.4 \%$ & $24.3 \%$ & $21.9 \%$ & $23.9 \%$ & $19.42 \%$ & $23.8 \%$ \\
\hline Body depth & $29.8 \%$ & $34.3 \%$ & $25.6 \%$ & $30.9 \%$ & $25.27 \%$ & $32.8 \%$ \\
\hline Body width & NA & NA & $10.6 \%$ & $24.3 \%$ & NA & NA \\
\hline $\begin{array}{l}\text { Caudal-peduncle } \\
\text { length }\end{array}$ & $8.7 \%$ & NA & $13.8 \%$ & $12.1 \%$ & NA & $10.6 \%$ \\
\hline $\begin{array}{l}\text { Caudal peduncle } \\
\text { depth }\end{array}$ & NA & NA & $5.0 \%$ & $3.7 \%$ & $3.72 \%$ & $5.6 \%$ \\
\hline $\begin{array}{l}\text { Height of anal fin } \\
\text { lobe }\end{array}$ & NA & NA & $8.8 \%$ & $7.3 \%$ & NA & $8.5 \%$ \\
\hline \multirow[t]{2}{*}{ Head length } & $24.3 \%$ & $24.3 \%$ & $23.8 \%$ & $24.2 \%$ & $23.38 \%$ & $25.5 \%$ \\
\hline & & & $\%$ Head Length & & & \\
\hline Pre Orbital length & $37.0 \%$ & $24.4 \%$ & $31.6 \%$ & $31.0 \%$ & $29.28 \%$ & $28.2 \%$ \\
\hline Post Orbital length & $45.0 \%$ & $48.8 \%$ & $60.5 \%$ & $60.5 \%$ & & $47.5 \%$ \\
\hline Eye diameter & $22.4 \%$ & $26.2 \%$ & $23.7 \%$ & $23.4 \%$ & $24.26 \%$ & $27.2 \%$ \\
\hline Inter Orbital length & NA & NA & $22.1 \%$ & $21.8 \%$ & $27.94 \%$ & NA \\
\hline 1st dorsal fin rays & VIII & VIII & VIII & VIII & VIII & VIII \\
\hline 2nd dorsal fin rays & I, 29 & $\mathrm{I}+28-33$ & $\mathrm{I}+24$ & $\mathrm{I}+29$ & $\mathrm{I}+31$ & $\mathrm{I}+29$ \\
\hline Pectoral fin rays & I, 19 & $\mathrm{I}-19$ & $\mathrm{I}+24$ & $\mathrm{I}+19$ & $\mathrm{I}+19$ & $I+19$ \\
\hline Pelvic fin rays & $\mathrm{I}, 5$ & $\mathrm{I}-5$ & $\mathrm{I}+5$ & $\mathrm{I}+5$ & $\mathrm{I}+5$ & $\mathrm{I}+5$ \\
\hline Anal fin rays & $\mathrm{II}, \mathrm{I}+19$ & $\mathrm{II}, \mathrm{I}+17-20$ & II, I+19 & II,I+19 & $\mathrm{II}+\mathrm{I}, 19$ & II, I+19 \\
\hline
\end{tabular}




\section{ACKNOWLEDGEMENTS}

This finding was obtained thanks to the European Data Collection Framework (DCF) module CampBiol - funded by European Union and the Italian Ministry for Agricultural, Food and Forestry Policies. We are also grateful to Dr. Fabio Fiorentino for many constructive comments and help, which greatly improved the manuscript.

\section{REFERENCES}

ANDALORO, F. \& A RINALDI. 1998. Fish biodiversity change in Mediterranean Sea as tropicalisation phenomenon indicator. In: G. Enne, M. D’Angelo \& C. Zanolla (Editors) Indicators for Assessing Desertification in the Mediterranean. ANPA \& Osservatorio Nazionale sulla Desertificazione. pp. 201-206.

ANDALORO F., M. FALAUTANO, M. SINOPOLI, F.M. PASSARELLI, C. PIPITONE, P. ADDIS, A. CAU \& L. CASTRIOTA. 2005. The lesser amberjack Seriola fasciata (Perciformes: Carangidae) in the Mediterranean: a recent colonist. Cybium, 29: 141-145.

ANONYMOUS. 2017. International bottom trawl survey in the Mediterranean. Instruction manual. Version 9. [MEDITS-handbook. Version n. 9.] MEDITS Working Group. 106 pp.

AZZURRO, E. \& F. ANDALORO. 2004. A new settled population of the lessepsian migrant Siganus luridus (Pisces: Siganidae) in Linosa IslandSicily Strait. J. Mar. Biol. Assoc. UK, 84: 819821. DOI:10.1017/ s0025315404009993h.

AZZURRO, E., S. SOTO, G. GAROFALO \& F. MAYNOU. 2013. Fistularia commersonii in the Mediterranean Sea: Invasion history and distribution modeling based on presence-only records. Biol. Invasions, 15: 977-990. DOI:10.1007/ s10530-012-0344-4.

AZZURRO, E., L. CASTRIOTA, M. FALAUTANO, F. GIARDINA \& F. ANDALORO. 2014. The silvercheeked toadfish Lagocephalus sceleratus (Gmelin, 1789) reaches Italian waters. J. Appl. Ichthyol., 30(5): 1050-1052. DOI: 10.1111/jai.12471
CORSINI, M., P. MARGIES, G. KONDILATOS \& P.E. ECONOMIDIS. 2006. Three new exotic fish records from the SE Aegean Greek waters. Sci. Mar., 70: 319-323. DOI:10.3989/ scimar.2006.70n2319

CARLTON, J.T. 1996. Biological invasions and cryptogenic species. Ecology, 77(6): 16531655. DOI:10.2307\%2F 2265767

CROCETTA, F., D. AGIUS, P. BALISTRERI, M. BARICHE, Y. BAYHAN, M. ÇAKIR, S. CIRIACO, M. CORSINI-FOKA, A. DEIDUN, R. EL ZRELLI, D. ERGÜDEN, J. EVANS, M. GHELIA, M. GIAVASI, P. KLEITOU, G. KONDYLATOS, L. LIPEJ, C. MIFSUD, Y. ÖZVAROL, A. PAGANO, P. PORTELLI, D. POURSANIDIS, L. RABAOUI, P. SCHEMBRI, E. TAŞKIN, F. TIRALONGO \& A. ZENETOS. 2015. New Mediterranean Biodiversity Records (October 2015). Med. Mar. Sci., 16(3): 682-702. DOI:10.12681/ mms. 1477

DEIDUN, A., L. CASTRIOTA \& S. ARRIGO. 2011. A tale of two Atlantic fish migrants: records of the lesser amberjack Seriola fasciata and the African hind Cephalopholis taeniops from the Maltese Islands. J. Black Sea/Medit. Environ., 17(3): 223-233.

DEIDUN, A., A. FENECH-FARRUGIA, L. CASTRIOTA, M. FALAUTANO, E. AZZURRO \& F. ANDALORO. 2015. First record of the silvercheeked toadfish Lagocephalus sceleratus (Gmelin, 1789) from Malta. BioInvasions Rec., 4:139-142. DOI:10.3391/ bir.2015.4.2.11.

DEIDUN, A., B. ZAVA, G. INSACCO \& M. CORSINIFOKA. 2018. First record of the Por's goatfish Upeneus pori (Actinopterygii, Perciformes, Mullidae) from Italian waters (western Ionian Sea). Acta Ichthyol. et Pisc., 48(1): 93-97. DOI:10.3750/AIEP/02269

DOĞDU, S. A., U. SAKALLI, M. GÜRLEK \& C. TURAN. 2019. The first record of the Lesser amberjack Seriola fasciata (Bloch, 1793) in the Çevlik coast of Turkey, Eastern Mediterranean Sea. Biharean Biologist, 13(1): 55-57.

FALSONE, F., D. SCANNELLA, M. L. GERACI, S. VITALE, G: SARDO F. \& FIORENTINO. 2020. Further records of Callinectes sapidus (Rathbun, 1896) (Decapoda, Brachyura, Portunidae) in the Strait of Sicily. Marine Biodiversity Records, 13(1): 1-6, https://doi.org/10.1186/ 
s41200-020-00190-5

FISCHER, W., G. BIANCHI \& W.B. SCOTT (Editors) 1981. FAO Species Identification Sheets for Fishery Purposes. Eastern Central Atlantic; Fishing Areas 34 and part of 47. Vol. 1 (in part). Canada Funds-in-Trust: Ottawa. pp. 326.

GALIL, B.S., A. MARCHINI, A. OCCHIPINTI-AMBROGI \& H. OJAVEER. 2017. The enlargement of the Suez Canal: Erythraean introductions and management challenges. Manag. Biol. Invasion, 8(2):141-152. DOI:10.3391/ mbi.2017.8.2.02

GERACI, M. L., M. DI LORENZO, F. FALSONE, D. SCANNELLA, F. DI MAIO, F. COLLOCA, S. VITALE \& F. SERENA. 2019. The occurrence of Norwegian skate, Dipturus nidarosiensis (Elasmobranchii: Rajiformes: Rajidae), in the Strait of Sicily, central Mediterranean. Acta Ichthyol. Piscat. 49 (2): 203-208. DOI: 10.3750/AIEP/02566

GOLANI, D., L. ORSI-RELINI, E. MASSUTI \& J.P. QUIGNARD. 2002. CIESM Atlas of exotic species in the Mediterranean. Vol. 1. Fishes. CIESM Publishers: Monaco. 256 pp.

GOULLETQUER, P., P. GROS, G. BOEUF \& J. WEBER. 2014. Biodiversity in the marine environment. Springer Science \& Business Media: Netherlands. 198 pp.

ISPRA. 2012. Identificazione e distribuzione nei mari italiani di specie non indigene URL: http://www.marinealien.sinanet.isprambiente.it/uploads/Seriola\%20fasciata.pdf (accessed 6.1.20).

JAWAD, L., A. MTAWEJ, A. IBRAHIM \& M. HASSAN. 2015. First record of the lesser amberjack Seriola fasciata (Teleostei: Carangidae) in Syrian coasts. Cah. Biol. Mar., 56(1): 81-84. DOI:10.21411/CBM.A.1337CE3E

KARA, M. H., E. BEN LAMINE, \& P. FRANCOUR. 2015. Range expansion of an invasive pufferfish, Lagocephalus sceleratus (Actinopterygii: Tetraodontiformes: Tetraodontidae), to the south-western Mediterranean. Acta Ichthyologica et Piscatoria, 45(1): 103-108. DOI:10.3750/aip2015.45.1.13

KAPIRIS, K., C. APOSTOLIDIS, R. BALDACCONI, N. BAŞUSTA, M. BILECENOGLU, G. BITARBOBORI,
D. BOYACI, Y. DIMITRIADIS, M. DJUROVIĆ, J. DULČIĆ, F. URUCAN, V. GEROVASILEIOU, M. GÖKOĞLU, D. KOUTSOUBAS, E. LEFKADITOU, L. LIPEJ, O. MARKOVIĆ, B. MAVRIČ, Y. ÖZVAROL, A. PESIC, V. PETRIKI, O. SIAPATIS, A. SINI, M. TIBULLO \& F. TIRALONGO. 2014. New Mediterranean Biodiversity Records (April, 2014). Med. Mar. Sci., 15(1): 198212. DOI: $10.12681 / \mathrm{mms} .737$

KARACHLE, P., A. ANGELIDIS, G. APOSTOLOPOULOS, D. AYAS, M. BALLESTEROS, C. BONNICI, M. BRODERSEN, L. CASTRIOTA, N. CHALARI, J. COTTAlORDA, F. CROCETTA, A. DEIDUN, Ž. ĐOĐO, A. DOGRAMMATZI, J. DULČIĆ, F. FIORENTINO, O. GÖNÜLAL, J. HARMELIN, G. INSACCO, D. IZQUIERDO-GÓMEZ, A. IZQUIERDO-MUÑOZ, A. JOKSIMOVIĆ, S. KAVADAS, M. MALAQUIAS, E. MADRENAS, D. MASSI, P. MICARELLI, D. MINCHIN, U. ÖNAL, P. OVALIS, D. POURSANIDIS, A. SIAPATIS, E. SPERONE, A. SPINELLI, C. STAMOULI, F. TIRALONGO, S. TUNÇER, D. YAGLIOGLU, B. ZAVA \& A. ZENETOS. 2016. New Mediterranean Biodiversity Records (March 2016). Med. Mar. Sci., 17(1): 230-252. DOI: 10.12681/mms. 1684

LOCKWOOD, J.L., M.F. HOOPES, \& M.P MARCHETTI. 2013. Invasion ecology: II edition. John Wiley \& Sons: United States. 456 pp.

LIPEJ, L., I. ACEVEDO, E. AKEL, A. ANASTASOPOULOU, A. ANGELIDIS, E. AZZURRO, L. CASTRIOTA, M. ÇELIK, L. CILENTI, F. CROCETTA, A. DEIDUN, A. DOGRAMMATZI, M. FALAUTANO, F. FERNÁNDEZ-ÁLVAREZ, R. GENNAIO, G. INSACCO, S. KATSANEVAKIS, J. LANGENECK, B. LOMBARDO, G. MANCINELLI, C. MYTILINEOU, L. PAPA, V. PITACCO, M. PONTES, D. POURSANIDIS, E.PRATO, S. RIZKALLA, P. RODRÍGUEZFLORES, C. STAMOULI, J. TEMPESTI, F. TIRALONGO, S. TIRNETTA, K. TSIRINTANIS, C. TURAN, D. YAGLIOGLU, G. ZAMINOS \& B. ZAVA. 2018. "New Mediterranean Biodiversity Records" (March 2017). Mediterranean Marine Science, 18(1), 179-201. DOI: 10.12681/mms.2068

MASSUTI, E. \& STEFANESCU C. 1993. First record of Seriola fasciata (Bloch, 1793) (Osteichthyes: Carangidae) in the Mediterranean. J. Fish Biol., 42(1): 143-144. 
DOI:10.1111/j.1095-8649.1993.tb00312.x

QUIGNARD, J.P. \& J.A. TOMASINI. 2000. Mediterranean fish biodiversity. Biol. Mar. Mediterr., 7(3): 1-66.

RUEDEN, C. T., SCHINDELIN, J., HINER, M. C., DEZONIA, B. E., WALTER, A. E., ARENA, E. T., \& ELICEIRI, K. W. 2017. ImageJ2: ImageJ for the next generation of scientific image data. BMC Bioinformatics, 18(1). doi:10.1186/ s12859-017-1934-Z

SCANNELLA, D., F. FALSONE, M.L. GERACI, C. FROGLIA, F. FIORENTINO, G.B. GIUSTO, B. ZAVA, G. INSACCO \& F. COLLOCA. 2017. Westward expansion of the Northern brown shrimp Penaeus aztecus Ives, 1891 (Crustacea, Penaeidae) in the Mediterranean Sea: First records in the Strait of Sicily. BioInvasion Rec. 6(1): 67-72. DOI:10.3391/ bir.2017.6.1.11

SERVELLO, G., F. ANDALORO, E. AZZURRO, L. CASTRIOTA, M. CATRA, A. CHIARORE, F. CROCETTA, M. D'ALESSANDRO, F. DENITTO, C. FROGLIA, C. GRAVILI, M. LANGER, S. LO BRUTTO, F. MASTROTOTARO, A. PETROCELLI, C. PIPITONE, S. PIRAINO, G. RELINI, D. SERIO, N. XENTIDIS \& A. ZENETOS. 2019. Marine alien species in Italy: A contribution to the implementation of descriptor D2 of the marine strategy framework directive. Mediterr. Mar. Sci., 0: 1-48. DOI: $10.12681 / \mathrm{mms} .18711$

SHAKMAN, E.A., A.B. ABDALHA, F. THALA, A. ALFATURI \& M. BARICHE. 2017. First records of seven marine organisms of different origins from Libya (Mediterranean Sea). BioInvasions Rec., 6(4): 377-382. DOI: 10.3391/ bir.2017.6.4.13

SONIN, O., P. SALAMEH \& D. GOLANI. 2009. First record of the lesser amberjack, Seriola fasciata (Actinopterygii: Perciformes: Carangidae), in the Levant. Acta Ichthyol. et Pisc., 39(1): 71-73. DOI:10.3750/aip2009.39.1.15
STAMOULI, C., E.K. AKEL, E. AZZURRO, R. BAKIU, A.A BAS, G. BITAR, Y.Ö. BOYACI, M. CAKALLI, M. CORSINI-FOKA, F. CROCETTA, B. DRAGIČEVIĆ, J. DULČIĆ, F. DURUCAN, R. EL ZRELLI, D. ERGUDEN, H. FILIZ, F. GIARDINA, I. GIOVOS, O. GÖNÜLAL, F. HEMIDA, A KASSAR, G. KONDYLATOS, A. MACALI, E. MANCINI, P. OVALIS, F. PALADINI DE MENDOZ, M. PAVIČIĆ, L. RABAOUI, S.I. RIZKALLA, F. TIRALONGO, C. TURAN, D. VRDOLJAK, S. YAPICI \& A. ZENETOS. 2017. New Mediterranean Biodiversity Records. Med. Mar. Sci., 18(3): 534-556. DOI: $10.12681 / \mathrm{mms} .15823$

TIRAlONGO, F., D. TIBULlO, G. MESSINA \& B.M. LOMBARDO. 2018. New records of two carangid species from the south-east coast of Sicily (Ionian Sea) and considerations about their presence and abundance. Acta Adriat., 59(2): 225-230. DOI:10.32582/aa.59.2.8.

VAVASIS, C., G. SIMOTAS, E. SPINOS, E.KONSTANTINIDIS, S. MINOUDI, A. TRIANTAFYLLIDIS \& C. PERDIKARIS. 2019. Occurrence of Pterois miles in the Island of Kefalonia (Greece): the Northernmost Dispersal Record in the Mediterranean Sea. Thalassas: An International Journal of Marine Sciences, 36(1): 171-175. DOI:10.1007/s41208-01900175-X

VITAle, S., M. ARCUlEO, A. VAZ, G.B. GIUSTO, S. GANCITANO \& S. RAGONESE. 2016. Otolithbased age and growth of the Lessepsian species Fistularia commersonii (Osteichtyes: Fistulariidae) in South of Sicily (Central Mediterranean Sea). It. J. Zool., 83: 490-496. DOI:10.1080/11250003.2016.1223759.

YAPICI, S. \& H. FILIZ. 2020. First occurrence of a lesser amberjack Seriola fasciata (Bloch, 1793) in the Aegean coasts of Turkey with morphological and molecular identification. Reg. Stud. Mar. Sci. DOI: 10.1016/j. rsma.2020.101494.

Received: 26 August 2019

Accepted:16 October 2020 


\title{
Dodatni nalaz rasprostranjenosti Seriola fasciata (Carangidae) u Sredozemnom moru
}

\author{
Michele Luca GERACI, Fabio FALSONE*, Danilo SCANNELLA i Sergio VITALE \\ *Kontakt, e-pošta: fabio.falsone@irbim.cnr.it
}

\begin{abstract}
SAŽETAK
U ovoj je bilješci opisan dodatni nalaz o vrsti Seriola fasciata s južne obale Sicilije (Sredozemno more). Ovaj nalaz je prvi na tom području i potvrđuje ključnu ulogu područja za širenje stranih vrsta (NIS).

Osim toga, prikazana je ažurirana karta prostorne raspodjele vrste Seriola fasciata, kao i rasprava o mogućoj pogrešnoj identifikaciji i kompeticiji s vrstom Seriola dumerili.
\end{abstract}

Ključne riječi: Herkulske vrste, Seriola fasciata, biološka raznolikost, prostorna rasprostranjenost, Sicilijski tjesnac 